\title{
Sistema videowall de bajo coste basado en Raspberry Pi, personalizable y configurable dinámica y remotamente vía Web
}

\author{
Pau Salvador, Fernando Boronat, Mario Montagud, Dani Marfil \\ Departamento de Comunicaciones, Immersive Interactive Media R\&D (IIM) Group, \\ Universitat Politècnica de València - Campus de Gandia \\ Calle Paraninf, 1, 46730, Grao de Gandia, Valencia (SPAIN) \\ \{pasallla@epsg, fboronat@dcom,mamontor@,damarre@dcom\}.upv.es
}

\begin{abstract}
Resumen- En este artículo se presenta una propuesta de sistema videowall, tanto con distribución uniforme como disforme, formado por dispositivos de bajo coste, personalizable y configurable dinámica y remotamente vía Web. Se han analizado los sistemas de videowall existentes y las diferentes posibilidades en cuanto a su desarrollo con dispositivos de bajo coste. Se ha optado por la utilización de Raspberry Pi como dispositivo hardware y OMXPlayer como software reproductor multimedia. La sincronización entre los diferentes reproductores se consigue mediante el uso de una señal de reloj común y el intercambio de mensajes de control entre dispositivos, así como adoptando técnicas (agresivas y suavizadas) de ajuste de los procesos de reproducción. El sistema incluye una interface web muy amigable que facilita su gestión y control de manera remota.
\end{abstract}

Palabras Clave- videowall, Raspberry Pi, OMXPlayer, NTP

\section{INTRODUCCIÓN}

Un videowall consiste en un conjunto de múltiples pantallas (monitores de PC, videoproyectores, paneles LED o TVs) que reproduce contenido multimedia de manera sincronizada, simulando una pantalla de mayores dimensiones. Entre las utilidades de los videowalls se pueden citar la creación de pantallas gigantes, paneles publicitarios (aplicación comercial), paneles informativos (p. ej. información sobre salidas y llegadas en aeropuertos, estadísticas...), etc. Su uso está muy extendido, entre muchos otros ejemplos, en escaparates, centros comerciales, aeropuertos y cartelería digital.

Las pantallas del videowall pueden estar distribuidas de forma uniforme, es decir, pantallas de idéntico tamaño distribuidas de forma matricial, bien formando un muro plano o bien curvo (en configuración $\mathrm{NxM}$, con $\mathrm{N}$ filas de $\mathrm{M}$ pantallas cada una), o bien de forma disforme, con pantallas de igual o diferentes tamaños y en diferentes orientaciones, para mostrar uno o varios elementos multimedia, como imágenes, vídeos, etc. (ver Fig. 1).

Aunque en el mercado ya existen numerosas soluciones de videowall comerciales, la mayoría requieren de hardware (HW) especial y software (SW) propietario, lo cuál las convierte en soluciones de coste muy elevado. Como ejemplos, se pueden citar los sistemas de Userful ${ }^{1}$, MagicInfo de Samsung ${ }^{2}$ y el sistema de $\mathrm{LG}^{3}$. Además, la mayoría de soluciones están basadas en la utilización de controladores del videowall o servidores especializados. Los precios suelen ser bastante altos, la gestión suele ser en local, y la configuración y expansion de los mismos por parte de los usuarios finales es prácticamente imposible.

\footnotetext{
${ }^{1}$ https://www.userful.com/ (último acceso: mayo 2017)

http://www.samsung.com/es/business/solutions-services/smartsignage-solutions/smart-signage-solutions/magicinfo-videowall (último acceso: mayo 2017)
} 

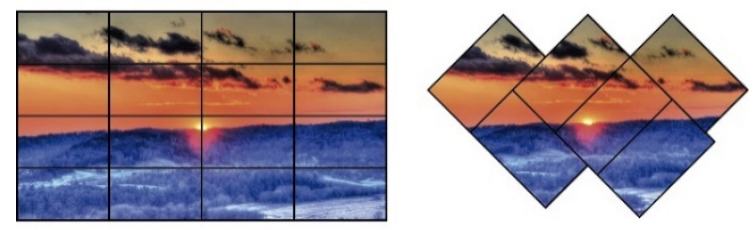

Fig. 1. Videowall uniforme plano (izquierda) y disforme (derecha)

En este artículo, se presenta la arquitectura, tanto HW como SW, de una solución económica ${ }^{4}$ para constituir un sistema de videowall formado por pantallas distribuidas, ya sea de forma uniforme o disforme, versátil, personalizable, y configurable dinámicamente y de manera remota vía IP, mediante una interfaz web. Su desarrollo se ha basado en el uso de dispositivos de bajo coste, Raspberry Pi (RPi en adelante), y se controla a través de una aplicación web, sin necesidad de instalar ninguna aplicación de control determinada, específica para cada plataforma o dispositivo.

Las pantallas del videowall se pueden combinar de varias formas: 1) todas las pantallas formando una única pantalla de dimensiones máximas, reproduciendo cada una una porción del mismo contenido (ver Fig. 1); 2) las pantallas se pueden agrupar de manera personalizable para diferentes contenidos formando secciones (Fig. 2).

En cada una de dichas pantallas, la imagen de vídeo correspondiente debe ser reproducida de forma perfectamente sincronizada con el resto. En caso contrario, una diferencia de unos pocos fotogramas entre las distintas pantallas de la misma sección del videowall puede ser notable y molesta (especialmente, cuando hay un cambio de plano o de escena), ya que las pantallas están muy cercanas o pegadas unas a otras.

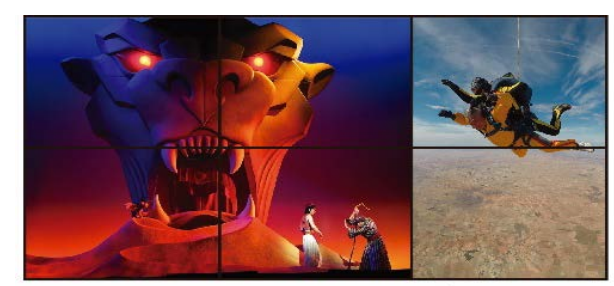

Fig. 2. Videowall 2x3 con dos secciones (2x2 y 2x1)

En el videowall propuesto, la reproducción de la parte del vídeo representada en cada pantalla está controlada por un dispositivo electrónico independiente, ejecutando un SW reproductor multimedia (player) independiente. Cada dispositivo reproduce el contenido con una referencia de reloj independiente, $y$, por tanto, de manera distinta, ya que los relojes, aunque tengan la misma frecuencia nominal, generalmente presentan diferentes grados de precisión y desviación de la misma. Con el objetivo de conseguir el efecto de videowall, con todas las partes del vídeo siendo reproducidas de forma sincronizada, se deberá establecer alguna solución de sincronización para conseguir que la reproducción de las

\footnotetext{
${ }^{4}$ Los costes de los sistemas comerciales rondan los $26.000 €$ (LG) y los
} $15.000 €$ (MagicInfo y Userful) para videowall disforme de 3x3 con distintas partes del contenido por los distintos dispositivos y pantallas esté completamente sincronizada. En la sección IV, se presenta la solución adoptada en el videowall propuesto.

El artículo está estructurado de la siguiente manera. La sección II recopila trabajos anteriores relacionados con la creación de videowalls. En la sección III se describen las configuraciones HW y SW del sistema videowall propuesto. En la sección IV, se analizan las diferentes posibilidades de sincronización de las pantallas del videowall, con sus ventajas e inconvenientes. La sección $\mathrm{V}$ presenta la aplicación web para la gestión y control del videowall. Por último, el artículo finaliza con la sección VI, con las conclusiones y líneas de trabajo futuro.

\section{TRABAJOS RELACIONADOS}

En esta sección, se revisan las tecnologías para el desarrollo de videowalls, las ventajas e inconvenientes de las implementaciones de videowall existentes, así omo una comparativa cualitativa de las mismas con la propuesta presentada. Además, se presentan algunos trabajos relacionados y se comparan con el presentado.

Actualmente, existen tres opciones en cuanto a las tecnologías de acceso y distribución de contenidos multimedia para ser reproducidos en las pantallas que forman el videowall (Fig. 3): 1) distribuir el contenido multimedia sobre estándares de distribución HW de video (normalmente, basados bien en splitters o divisores de señal de vídeo, o bien en pantallas especiales que se conectan entre sí); 2) distribuir el contenido por streaming IP; o 3) acceder directamente a contenido almacenado en una unidad de disco compartida en red.

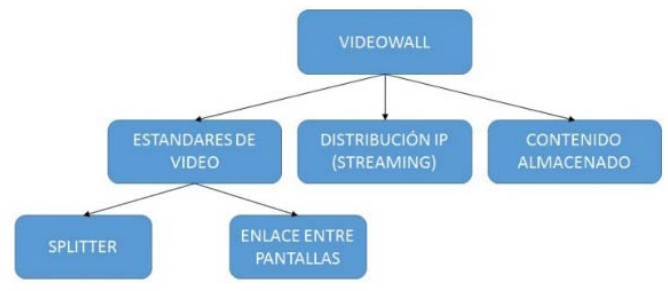

Fig. 3. Tecnologías videowall

Con respecto a la primera opción, por un lado, se pueden utilizar splitters de vídeo que son dispositivos activos que tienen una entrada y varias salidas de vídeo. Por la entrada se recibe el contenido del vídeo con la resolución completa a ser reproducido en el videowall, y el propio dispositivo se encarga de dividir dicho vídeo (sus imágenes) en diferentes partes (o no, si se desea que todas las pantallas reproduzcan exactamente el mismo contenido) que distribuirá a cada una de sus salidas a las que se conectarán las diferentes pantallas que forman el videowall. Esta opción está pensada para casos en los que todas las pantallas sean iguales y con configuraciones

pantallas de 46” y el sistema propuesto con RPi tendría un coste de $9.500 €$. 
sencillas. Tiene la ventaja de que es simple de instalar, pero tiene los inconvenientes de ser una solución poco escalable, se necesita HW específico, la fuente de contenido debe estar próxima a las pantallas y las opciones de configuración son bastante limitadas.

Por otro lado, dentro de la primera opción mencionada, también cabe la posibilidad de enlazar las pantallas para formar videowalls. Se basa en sistemas propietarios creados por determinadas empresas fabricantes de pantallas (ej. LG o NEC), que permiten que las pantallas se puedan enlazar entre ellas (por ejemplo, a través del DisplayPort en los sistemas NEC) para utilizarlas como un videowall. Esta opción tiene la ventaja de ser escalable (el sistema de NEC admite hasta 100 pantallas, en una matriz 10x10), ya que sólo hay que ir cableando las diferentes pantallas en serie. Sin embargo, tiene varios inconvenientes, como el coste elevado y el hecho de que todas las pantallas han de ser del mismo fabricante. Además, existe un problema asociado con el mantenimiento, ya que posibles futuras averías de pantallas implica sustituirlas por pantallas del mismo fabricante y, probablemente, modelo.

Con respecto a la segunda opción, basada en la distribución de los contenidos vía IP (streaming), cabe resaltar que es escalable y adaptable (p.ej., Samsung dispone de la solución MagicInfo que admite hasta 250 pantallas de gran formato), a la vez que muy simple en cuanto a instalación física se refiere, ya que sólo requiere la interconexión de todos los dispositivos mediante una red IP local. Sin embargo, será necesaria la disponibilidad de una red que proporcione el ancho de banda suficiente para poder distribuir los diferentes contenidos al mismo tiempo a las diferentes pantallas. También se requiere el uso de un ordenador potente que se encargue de dividr el vídeo en varios flujos (uno por cada pantalla) y realice el envío (streaming) vía IP del contenido correspondiente a ser reproducido en cada pantalla, especialmente cuando se realizan varios envíos simultáneos. La necesidad de dicho ordenador con suficientes recursos encarece el sistema. Además, para cada pantalla se requiere de HW y SW específicos (p.ej., dispositivos denominados Zero clients o RPi, con SW capaz de decodificar y reproducir el vídeo recibido por streaming) para decodificar el vídeo y entregarlos a los interfaces de salida o pantalla (por HDMI, por ejemplo). Con el sistema de distribución del contenido por IP podemos encontrar el sistema de Samsung nombrado anteriormente y el definido en [1].

La tercera opción indicada permite prescindir de dicho ordenador potente $y$, por tanto, abaratar el coste del videowall, si en cada pantalla se coloca un dispositivo de bajo coste que accede a contenido almacenado en una unidad de disco compartida. En dicho caso, cada dispositivo o bien un dispositivo de control se puede encargar de seleccionar la/s parte/s del vídeo a reproducir, sin necesidad de realizar streaming. De esta forma, se puede crear un videowall con dispositivos económicos y con menos recursos. En cuanto a inconvenientes, en este caso también se necesita una red que proporcione el suficiente ancho de banda para poder realizar los diferentes accesos simultáneos al contenido almacenado.

Por un lado, en el videowall propuesto se desea que tanto la configuración del contenido a reproducir en las diferentes secciones del videowall como la configuración del número de pantallas que componen las mismas sea dinámica y controlable remotamente. Por tanto, la primera opción de utilizar estándares de vídeo no ofrece la flexibilidad deseada y, por tanto, fue descartada desde el principio. Por otro lado, hoy en día existe electrónica de red de bajo coste que proporciona conectividad LAN con velocidad suficiente para trabajar con contenido almacenado, así como para cargar y descargar contenido a una unidad de disco compartida en red durante el funcionamiento del videowall, sin necesidad de implementar un servidor de streaming, lo cual encarecería el sistema. Es por ello que se ha optado, en esta primera versión, por la tercera opción, basada en el uso de contenido almacenado y de dispositivos RPi.

Se han encontado varias propuestas de videowalls basadas en RPi y su reproductor OMXPlayer: [1], [2] y [3], que se han mejorado en el presente trabajo. Dichas propuestas no satisfacen las necesidades mínimas para el videowall requerido, por tanto, se procede a realizar un desarrollo completo del sistema. La Tabla I presenta una comparativa resumida entre dichas propuestas y la presentada en este artículo.

Tabla I

COMPARATIVA TRABAJOS RELACIONADOS

\begin{tabular}{|c|c|c|c|c|}
\hline & {$[2]$} & [3] & [1] & $\begin{array}{c}\text { Sistema } \\
\text { Propuesto }\end{array}$ \\
\hline Programaciones & No & $\mathrm{NS} / \mathrm{NC}$ & $\mathrm{NS} / \mathrm{NC}$ & $\mathrm{Si}$ \\
\hline Playlist & No & $\mathrm{NS} / \mathrm{NC}$ & $\mathrm{NS} / \mathrm{NC}$ & $\mathrm{Si}$ \\
\hline $\begin{array}{c}\text { Diferentes } \\
\text { secciones }\end{array}$ & No & $\mathrm{NS} / \mathrm{NC}$ & $\mathrm{NS} / \mathrm{NC}$ & $\mathrm{Si}$ \\
\hline $\begin{array}{l}\text { Videowall } \\
\text { disforme }\end{array}$ & $\mathrm{Si}$ & $\mathrm{Si}$ & No & $\mathrm{Si}$ \\
\hline Rotar pantallas & No & No & No & $\mathrm{Si}$ \\
\hline $\begin{array}{c}\text { Slideshow de } \\
\text { imágenes }\end{array}$ & No & No & No & $\mathrm{Si}$ \\
\hline $\begin{array}{c}\text { Sistema de } \\
\text { sincronía }\end{array}$ & $\mathrm{Si}$ & $\mathrm{Si}$ & $\mathrm{Si}$ & $\mathrm{Si}$ \\
\hline $\begin{array}{l}\text { Configuración } \\
\text { dinámica }\end{array}$ & No & No & $\mathrm{Si}$ & $\mathrm{Si}$ \\
\hline $\begin{array}{c}\text { Configuración } \\
\text { vía web }\end{array}$ & No & No & $\mathrm{Si}$ & $\mathrm{Si}$ \\
\hline
\end{tabular}

\section{Desarrollo Del Sistema Videowall}

En esta sección se presenta toda la arquitectura HW y SW que se ha realizado para el desarrollo del sistema videowall, personalizable, controlable dinámica y remotamente vía web.

\section{A. Funcionalidad}

En la arquitectura propuesta se puede seleccionar cualquier combinación de pantallas, ya sea uniforme o disforme (incluso incluyendo pantallas con rotación) y formar grupos de pantallas con diferentes contenidos dentro del videowall completo (véase la Fig. 2). A estos grupos o combinaciones de pantallas se las denomina secciones del videowall, en adelante, en este documento. 
Mediante una aplicación web (SW multi-plataforma) de control se puede gestionar y configurar el videowall, seleccionando el contenido a mostrar en cada una de las pantallas del mismo, sea cual sea su distribución. Además, la aplicación de gestión dispone de la posibilidad de programar las reproducciones (horarios de reproducción) de cada sección del videowall, reproducir playlists, poner la reproducción de los vídeos en bucle, así como controlar qué audio o audios se escucharán por los altavoces del videowall.

Por lo que respecta a los contenidos, como se ha comentado previamente, se almacenarán en un disco duro externo accesible a través de la red local por los dispositivos del videowall (RPi).

\section{B. Hardware}

Para el control y la gestión del videowall, se van a utilizar dispositivos RPi, en concreto, la Raspberry Pi 3 Modelo $\mathrm{B}^{5}$. Serán necesarios tantos como pantallas tenga el videowall, más uno adicional que se encargará del control de todo el sistema y de la comunicación con el dispositivo utilizado por el usuario para la gestión vía web. En la Fig. 4 se puede ver un ejemplo de videowall formado por una combinación 2x3. Para dicha configuración, el HW del sistema está formado por seis pantallas, altavoces, siete RPi, un disco duro externo USB 3.0, un switch Fast-Ethernet, y un dispositivo externo (móvil, tablet, PC, portátil...) con un navegador y conectividad IP.

Excepto la de control, cada RPi se conectará por HDMI a su pantalla correspondiente. Todas las RPi estarán conectadas en LAN a través de un switch a 100 Mbits/s. En este caso, la RPi de control y el dispositivo de usuario deberán tener conectividad IP (p. ej., de forma inalámbrica a través de un punto de acceso Wifi externo al videowall, preferiblemente, con conexión a Internet, para facilitar su configuración de forma remota).

A nivel de la red LAN, todos los dispositivos estarán configurados con direcciones IP estáticas de la red IP 192.168.0.0/16. En su conexión con esta red, a la RPi de control del videowall se le asignará siempre la IP 192.168.0.1/16. La RPi de control estará conectada a dos redes, la LAN 192.168.0.0/16 conectada a través del cable de Ethernet y también a una red (p. ej., Wifi) externa si se desea que el videowall pueda pueda ser configurado remotamente. La configuración IP de la conexión externa podrá ser estática o conseguirla a través de DHCP y permitirá la gestión remota del videowall.

Por un lado, en el caso de videowalls con distribución uniforme, para facilitar la gestión, aunque se pueda asignar cualquier dirección IP de forma dinámica y controlar las asignaciones mediante tablas, a cada RPi conectada a un monitor se ha decidido asignarle la dirección IP/máscara de red 192.168.x.y/16, siendo $x$ el

${ }^{5}$ La Raspberry Pi 3 Modelo B es un microcomputador de bajo coste que ofrece, entre otras, las siguientes prestaciones: Tarjeta 100 Base-T (Fast Ethernet), Wifi 802.11n, SW de código abierto y decodificación por HW de contenido H264 con una resolución de 1080p, con una tasa número de fila (siendo la fila superior la fila 1) e y el número de columna (siendo la columna de la izquierda la número 1), tal y como se muestra en la Fig. 4. Por otro lado, en el caso de videowalls con distribución disforme, la asignación de direcciones IP puede seguir cualquier lógica y quedará registrada en la base de datos del videowall. En este caso, se ha decidido numerar las pantallas y registrar en una tabla la asignación de direcciones a cada una de ellas.

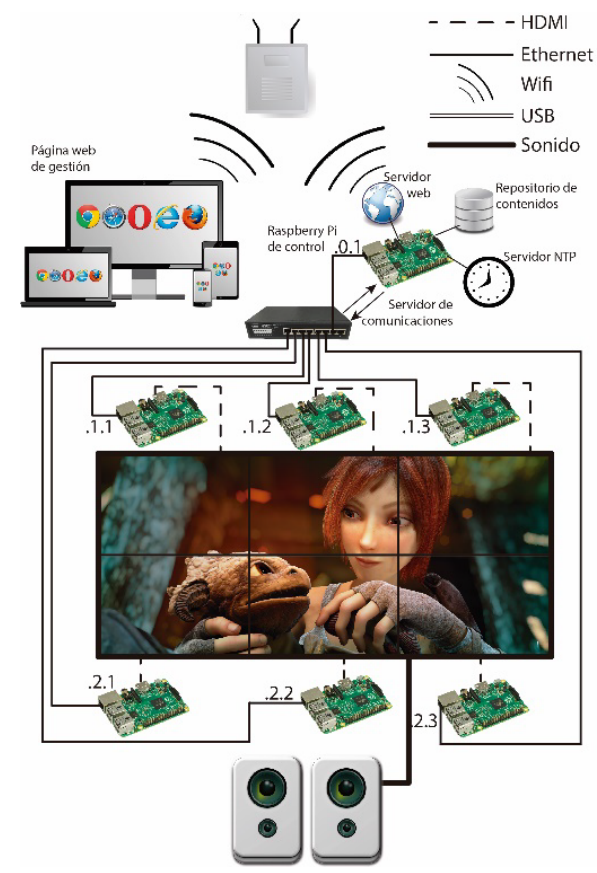

Fig. 4. Configuración del escenario

Para realizar un control de los diferentes flujos de audio a ser reproducidos por los altavoces del videowall, se ha diseñado un circuito electrónico para cada canal (Left/Right), que se muestra en la Fig. 5.

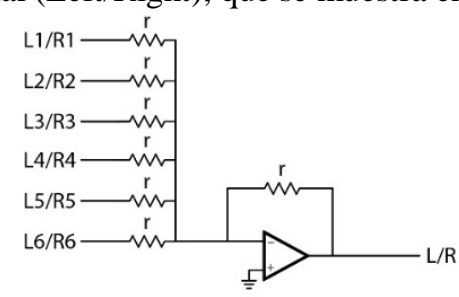

Fig. 5. Circuito electrónico del audio

Se puede apreciar que está formado por dos sumadores con amplificadores operacionales. En cada sumador están las seis salidas de cada canal de audio (L o R, respectivamente) de las RPi (L1 a L6 o R1 a R6), así como la entrada de los altavoces ( $\mathrm{L}$ y R). Cada circuito cuenta con siete resistencias (r) en el sumador de cada canal, cuyo valor es indiferente. Todas las resistencias de cada sumador deben ser iguales para no amplificar ni de 30 imágenes por segundo. Proyecto Raspberry. https://www.raspberrypi.org/ (último acceso mayo 2017) 
atenuar las señales de audio. A través del SW de gestión del videowall, se pueden seleccionar los audios que se quieren reproducir y estos se sumarán en el circuito descrito.

\section{Software}

\section{C.1. Sistema Operativo}

Todas las RPi empleadas incorporan Raspbian ${ }^{6}$ como sistema operativo (S.O.). Está basado en Debian, pero simplificado para estos dispositivos.

\section{C.2. Servidor de Contenidos}

En la RPi de control se ha habilitado un servidor FTP para el acceso a la unidad de disco USB compartida, en el que se han configurado los permisos adecuados para cada tipo de usuario. De esta forma, cada usuario sólo puede acceder a determinadas carpetas, y subir y/o seleccionar contenido para ser mostrado en las diferentes secciones del videowall.

\section{C.3. Reproductor de vídeo}

A la hora de escoger el reproductor de vídeo a ejecutar en las RPi, se tuvieron en cuenta dos opciones que incluyeran o permitieran implementar soluciones de sincronización entre la reproducción de diferentes instancias del mismo en diferentes dispositivos, conectados a través de redes IP. Las dos opciones fueron la plataforma Gstreamer [4] (incluyendo los elementos para la reproducción y sincronización) y el reproductor OMXPlayer [5].

Al principio, se instaló en las RPis el framework Gstreamer [4] y se intentó utilizar su reproductor, puesto que GStreamer también soporta de manera nativa una solución de sincronización multimedia entre dispositivos, ya utilizada por nuestro grupo en otros proyectos, en dispositivos basados en Linux. Se comprobó que el rendimiento de reproducción no era el deseado, y, por tanto, no fue posible reproducir contenido multimedia de manera correcta.

Sin embargo, el reproductor OMXPlayer [5] funcionó a la perfección $y$, por tanto, fue el escogido para el videowall propuesto. Dicho reproductor está específicamente creado para la RPi, y aprovecha la decodificación por HW del propio dispositivo, de forma que libera mucho la carga del sistema S.O. Para ejecutar este reproductor, se ha utilizado una librería desarrollada para Python $^{7}$, que permite controlar su proceso de reproducción a través de un sistema de comunicación entre procesos, denominado D-Bus (Desktop Bus ${ }^{8}$ ).

OMXPlayer no contempla la opción de reproducción de videos con un ángulo de rotación diferente a 90, 180 o 270 grados. Es por ello que, como el videowall propuesto, cuando se le configura una distribución

6 Distribución Raspbian. Página de descarga: https://www.raspberrypi.org/downloads/raspbian/ (último acceso mayo 2017)

7 OMXPlayer para Pyhton. Repositorio oficial: https://github.com/willprice/python-omxplayer-wrapper (último acceso mayo 2017) disforme, permite colocar las pantallas en cualquier disposición, se ha implementado un script basado en la herramienta ffmpeg $^{9}$, que crea copias del contenido con el/los ángulo/s necesario/s. Dicho script se ejecuta tras la configuración de videowalls disformes con pantallas con inclinación, así como cada vez que se sube contenido a ser reproducido en ellas.

\section{C.4. Servidor de Tiempo Global}

Para conseguir la sincronización de la reproducción de las porciones de los vídeos correspondientes a cada pantalla de las secciones del videowall (es decir, entre los reproductores ejecutándose en cada una de las RPi), todos los procesos de reproducción deben disponer de una referencia de reloj global común. En el videowall propuesto, el reloj de todas las RPi estará sincronizado mediante un servidor NTP (Network Time Protocol, RFC 5905 [6]), instalado en la RPi de control. Se ha escogido la opción de NTP frente a PTP (Precission Time Protocol, estándar IEEE 1588), ya que la precisión que se puede conseguir en una LAN es más que suficiente para el videowall y no se requiere de HW específico.

\section{C.5. Servidor de Comunicaciones}

En la RPi de control se ha implementado un servidor de Websockets, basado en Node.js ${ }^{10}$ y las librerías Socket.IO, para permitir el intercambio de mensajes entre todas las RPis que forman el videowall. Cada RPi, al iniciarse, se conectará a dicho servidor de Websockets y quedará a la espera de recibir mensajes de control (de la RPi de control, según la programación o configuración del contenido del videowall realizada) o de sincronismo (explicados en la siguiente sección).

En cuanto a los mensajes de control, cada Rpi puede recibir de la RPi de control, de forma unicast, tres posibles mensajes de tipo texto, iniciados con las cadenas "VIDEO”, “AUDIO” o "IMAGEN", según el contenido a reproducirse en la pantalla asociada a dicha $\mathrm{RPi}$, seguidas de varios parámetros separados por '\%”:

\section{"VIDEO\%ruta\%X1\%Y1\%X2\%Y2\%posición\%tiempo\%master" "ACCION\%acción\%tiempo" \\ "IMAGEN\%ruta\%X1\%Y1\%X2\%Y2\%posición\%tiempo"}

En el primer mensaje, relativo a contenido de vídeo, el parámetro ruta contendrá la ruta del vídeo a reproducir; los parámetros $X 1$ e $Y 1$ se corresponden con las coordenadas $x$ e $y$ de la esquina superior izquierda de la porción de video que ha de reproducir; $X 2$ e $Y 2$ se corresponden con las coordenadas $x$ e $y$ de la esquina inferior derecha de la porción de video que ha de reproducir; posición es la posición donde debe ir centrado el vídeo a reproducir y puede tener uno de los siguientes valores: $\{L, R, U, D, C\}$ que equivalen a izquierda, derecha, arriba, abajo o centro, respectivamente, y sirve para

\footnotetext{
8 https://www.freedesktop.org/wiki/Software/dbus/ (último acceso mayo 2017)

9 https://ffmpeg.org/ (último acceso mayo 2017)

10 https://nodejs.org/es/ (último acceso mayo 2017)
} 
ajustar la posición de las porciones de los vídeos en las pantallas en casos especiales (pantallas periféricas del videowall o pantallas en distribución disforme); y tiempo contiene el instante en que ha de empezar a reproducirse el video. Este parámetro se ha incluido para conseguir que todas las pantallas inicien la reproducción en el mismo instante de tiempo NTP (sincronización inicial de la reproducción). El parámetro master será de tipo booleano e indicará a cada RPi si tiene rol maestro (master) o esclavo. Como ejemplo, en el videowall uniforme de la Fig. 4, el mensaje que recibiría la pantalla superior derecha (IP 192.168.1.3), si en todo el videowall (una única sección de 2x3 pantallas) se va a reproducir un vídeo de resolución 1920x1080, sería:

VIDEO\%ruta\%1440\%0\%1920\%540\%L\%tiempo\%false

En el segundo mensaje, relativo a la Acción a realizar, el parámetro acción puede contener los valores "play", "pause", "mute" o "unmute", para iniciar o pausar la reproducción, bloquear o desbloquear el audio, respectivamente; mientras que el parámetro tiempo contiene el instante NTP en el que ha de ejecutarse exactamente dicha acción.

En el tercer mensaje, relativo al contenido de imágenes, el parámetro ruta contendrá la ruta de la imagen a mostrar por pantalla; y los parametros $X 1, Y 1$, $X 2, Y 2$, posición y tiempo tienen el mismo significado anteriormente descrito para el primer mensaje para contenido de video. En este caso, al tratarse de mostrar una imagen estática en una sección del videowall (o en todo) no será necesario implementar mecanismos de sincronización de procesos reproductores. Tan sólo será necesario sincronizar el instante en el que todas las pantallas de la sección en la que vaya a aparecer una porción de la imagen empiecen a mostrarla en el mismo instante NTP.

Al recibir el primer o tercer mensaje, si la RPi ya está reproduciendo contenido diferente, detendrá dicha reproducción y empezará a reproducir el nuevo contenido en el instante indicado dejando alguna parte de la pantalla en negro si fuera necesario para respetar la relación de aspecto del vídeo o la imagen original.

\section{C.6. Servidor Web}

Se ha instalado un servidor web basado en node.js en la RPi de control, a través del cual se podrá gestionar todo el sistema. Los usuarios pueden acceder, a través de un navegador, a dicho servidor para realizar la gestión/configuración del videowall. La aplicación/interface web desarrollada se presenta en la sección $\mathrm{V}$.

\section{IV.SOLUCIÓN DE SINCRONIZACIÓN}

\section{A. Sincronización inicial}

Como se ha visto en los mensajes, cuando cada una de las RPi del videowall recibe una orden de la RPi de control, indicando la reproducción de contenido, esta incluye el instante NTP en el que ha de iniciarse dicha reproducción (para su cálculo se tiene en cuenta el retardo de las comunicaciones entre ellas). Por tanto, si todas las RPi tienen sus relojes sincronizados con el servidor NTP de la RPi de control, todas las pantallas de cada una de las secciones del videowall iniciarán su reproducción al mismo tiempo y de forma sincronizada.

\section{B. Sincronización fina durante la reproducción}

Los relojes de cada una de las RPi y las tasas de consumo de sus procesos de reproducción no tienen por qué ser exactamente iguales, sino que pueden sufrir desviaciones durante la sesión. Una vez iniciada la reproducción, dichas desviaciones pueden llevar, incluso en poco tiempo, a asincronías acumuladas que resulten en diferencias de un cierto número de fotogramas entre las distintas pantallas de la misma sección. Esto puede ser notable y molesto para los usuarios (especialmente en cambios de plano o de escena). Es por ello que se necesitan mecanismos de ajuste frecuente de los procesos de reproducción de las diferentes pantallas que conforman cada sección del videowall que permitan corregir dichas desviaciones.

En este caso, para sincronizar los puntos de reproducción de diferentes instancias del reproductor OMXPlayer se ha optado por utilizar la misma referencia de reloj global (proporcionada por NTP) para sincronizar el reloj local de cada una de las instancias del reproductor, así como utilizar el módulo pyOmxSync ${ }^{11}$, desarrollado en Python, realizándole algunas modificaciones. Dicho módulo permite la sincronización de los procesos de reproducción de diferentes instancias del reproductor OMXPlayer conectadas a través de redes IP, siguiendo un esquema de control maestro/esclavo. La RPi de una de las pantallas de cada sección del videowall se comporta como reproductor maestro (de referencia), en cuanto al proceso de sincronización, del resto de procesos de las RPis de las otras pantallas pertenecientes a esa misma sección. Aunque se puede cambiar en la configuración, por defecto, sea cual sea la distribución del videowall (unifrome o disforme), se escogerá como dispositivo maestro a la RPi con menor dirección IP de cada sección.

La RPi con rol de maestro de cada sección del videowall, envía, a través del servidor Websocket a todas las demás RPi de su misma sección, cada cierto tiempo configurable (por ejemplo, un segundo), su punto de reproducción del vídeo (posición respecto al principio del vídeo) y el instante global de tiempo NTP (timestamp) correspondiente a dicho punto de reproducción. Las demás RPis, configuradas como dispositivos esclavos de esa misma sección y que, por tanto, están reproduciendo el mismo vídeo, cuando reciben dicha información la utilizan para comparar su propio punto de reproducción con el del dispositivo maestro. Si la diferencia entre 
ambos supera un cierto margen o umbral de asincronía permitido (también configurable), corregirá su proceso de reproducción mediante ajustes, bien agresivos (saltos y pausas) o bien adaptativos o suaves (Adaptive Media Playout o AMP, [7]). Tras cada ajuste, y una vez sincronizado el proceso de reproducción, se esperarán un tiempo de guarda (configurable) para volver a sincronizar y dejar que, mientras, se estabilice dicho proceso. Durante el mismo hará caso omiso a los mensajes recibidos del maestro, en caso que los reciba.

Se ha comprobado en el laboratorio que con este método, en media, se puede mantener las asincronías por debajo de 60 ms. En https://goo.gl/PiFn5E se encuentra disponible un video mostrando la funcionalidad del sistema así como el grado de sincronización conseguida.

\section{APLICACIÓN DE GESTIÓN DEL VIDEOWALL}

En esta sección se presenta la aplicación web realizada para la gestión y configuración del videowall. Para este propósito se ha instalado un servidor web mediante Node.js en la RPi de control. La gestión del videowall se realiza conectándose a este servidor web mediante un navegador (vía http://IP_Rpi_de_control).

La aplicación de configuración tiene varias pantallas. En primer lugar, se muestra la pantalla de entrada al servidor, la página de inicio de sesión (Fig. 6). Se ha implementado un control de usuarios con diferentes roles para que cada uno de ellos tenga acceso a unas carpetas y archivos en concreto de la unidad compartida y sólo pueda actuar sobre ellos (gestionando el contenido a ser seleccionable por dicho usuario para ser reproducido en el videowall). También se ha dado de alta un usuario Admin con rol de administrador que podrá, además de administrar el videowall, añadir y eliminar usuarios.

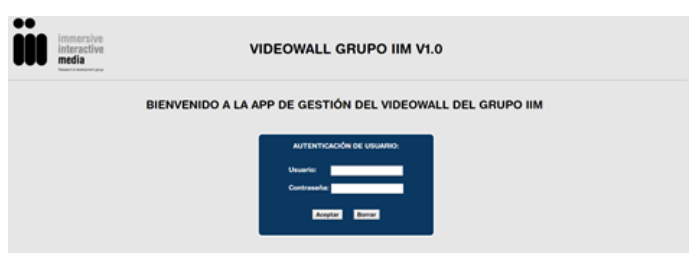

Fig. 6. Página de inicio de sesión

Una vez introducidas las credenciales, se pasa a la página mostrada en la Fig. 7, en la que aparecerán diferentes opciones de la aplicación web, dependiendo del rol de usuario que haya iniciado la sesión. Incluye las opciones de configuración general del videowall, gestión de usuarios y parámetros de red (todas ellas accesibles para el usuario Admin), y la de selección de contenido (accesible para todos los usuarios). La selección de cada una de las opciones lleva a una determinada página web para realizar la operación correspondiente.

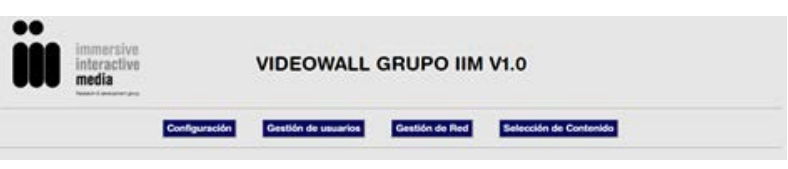

Fig. 7. Página con todas las opciones de configuración (Admin)
La Fig. 8 muestran las ventanas de configuración general del videowall (uniforme a la izquierda y disforme a la derecha) a la que sólo pueden acceder los usuarios con rol de administrador (Admin). Se permite seleccionar si se trata de un videowall uniforme o disforme, así como configurar los siguientes parámetros de sincronización: el intervalo de envío de información de sincronización por parte de los dispositivos maestros, el valor de la asinconía máxima permitida entre la reproducción de las diferentes pantallas del videowall, el intervalo de guarda y el tipo de ajuste de sincronización (agresiva o AMP).
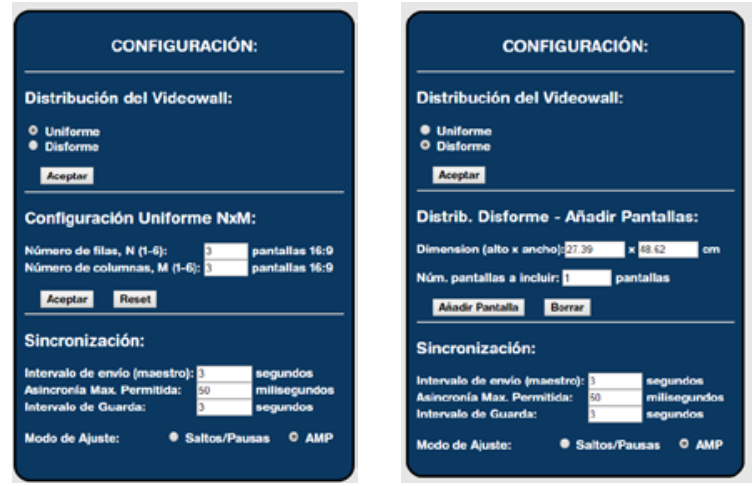

Fig. 8. Página de configuración general del videowall

La parte de la izquierda de la Fig.8 muestra la página de configuración del videowall en caso de tener una distribución de pantallas uniforme (matriz NxM). Permite seleccionar el número de pantallas en horizontal y el número de pantallas en vertical. La parte de la derecha de la Fig. 8 muestra la página de configuración del videowall en caso de tener una distribución de pantallas disforme. Se permite añadir pantallas al videowall, seleccionando su tamaño y número. Se crean en un panel y se permite arrastrarlas con el ratón, así como proporcionarles un ángulo de rotación para colocarlas, tal y como estarán las pantallas físicamente en el videowall real. Las pantallas se pueden seleccionar de forma independiente y cambiar sus propiedades (tamaño, posición y rotación).

La Fig. 9 muestra la página de gestión de usuarios, en la que se pueden crear, editar y eliminar usuarios, además de configurar los permisos de acceso a las carpetas correspondientes. A esta página sólo tendrán acceso los usuarios con rol de administrador (Admin). Al pulsar el botón de 'Añadir Nuevo Usuario', aparecerá la ventana de la parte inferior de la figura.

La Fig. 10 muestra la Gestión de la información de red de los dispositivos del videowall (sólo usuario Admin). Al pulsar el botón de 'Añadir Pantalla', aparecerá la ventana de la parte inferior de la figura.

La Fig. 11 muestra la página con la distribución del videowall (uniforme, en este caso) en la que se pueden seleccionar las pantallas que conforman cada sección del videowall, así como el contenido a mostrar en cada una de ellas. En caso de tratarse de un videowall uniforme, se deben seleccionar la pantalla de la esquina superior izquierda y la pantalla de la esquina inferior derecha de cada sección, e inmediatamente aparece una ventana para 
seleccionar el contenido que se quiere visualizar en dicha sección. Si el contenido que se pretende enviar contiene audio, se preguntará si se quiere reproducir con audio o sin él (opción muted). En el caso de un videowall disforme se deben seleccionar, una a una, las pantallas en las que se quiere mostrar el contenido y pulsar sobre el botón 'Seleccionar Vídeos/Imágenes' y aparecerá la ventana ya comentada para seleccionar el contenido a visualizar. En esta página también aparecen botones para reproducir/reanudar y pausar la reproducción, así como tres opciones a destacar: 'Programación', 'Seleccionar Audio', 'Cargar Configuración’ y 'Reset Videowall'.

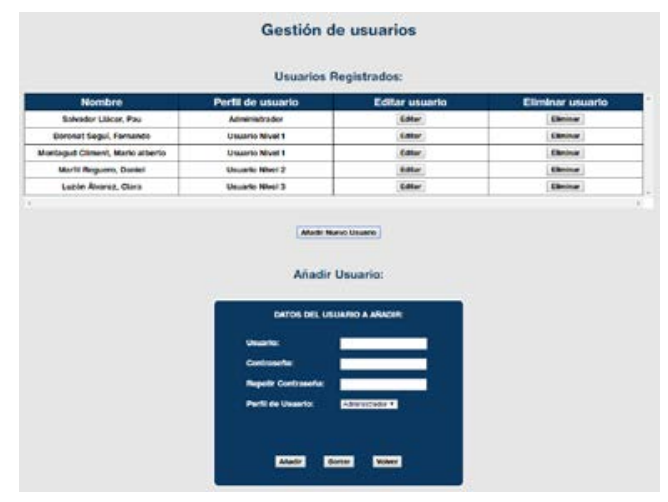

Fig. 9. Página de gestión de usuarios

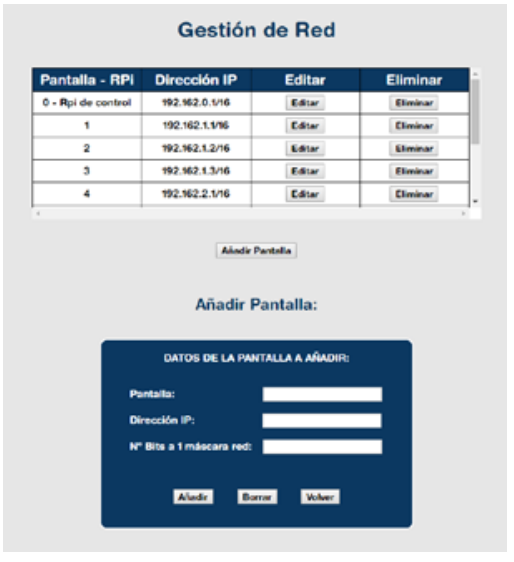

Fig. 10. Gestión de parámetros de red.

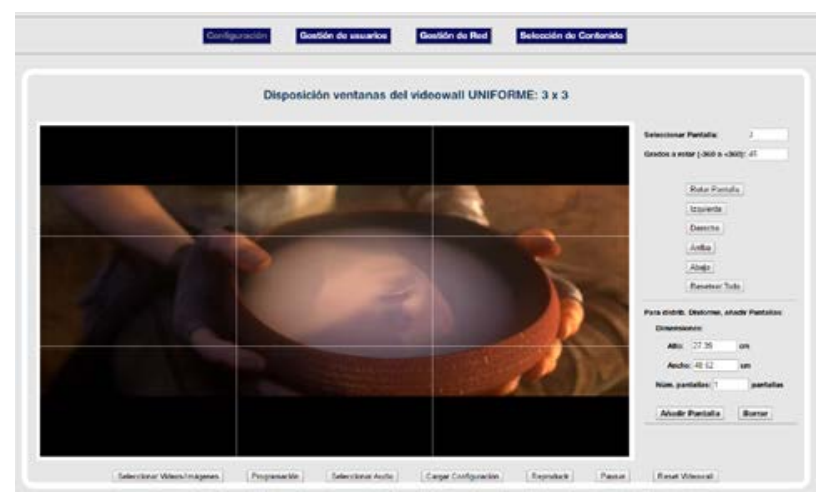

Fig. 10. Ventana de selección del contenido y programación

'Programación' permite guardar secuencias de reproducción programadas para cada sección del videowall. 'Seleccionar Audio' permite activar la reproducción de los canales asociados a cada contenido siendo reproducido en alguna sección del videowall.
Aparece la lista con los vídeos siendo reproducidos y, mediante controles de tipo checkbox, se puede seleccionar (o deseleccionar) cualquiera para que su audio empieze a sonar (o no) por los altavoces del videowall. 'Cargar Configuración' envía la configuración a la RPi de control que será la encargada de enviar los mensajes pertinentes a las RPis de cada pantalla. 'Reset Videowall' detiene la reproducción de las pantallas del videowall y borra las asignaciones de contenido a cada una de las secciones del mismo.

\section{CONCLUSIONES Y TRABAJO FUTURO}

En este artículo se ha presentado una propuesta HW y SW para diseño y configuración de sistemas videowalls, tanto uniformes como disformes, muy completo y flexible, controlable dinámicamente vía web y, lo más importante, utilizando dispositivos de muy bajo coste (cost-effective). Tras analizar las diferentes opciones existentes para el desarrollo de videowalls, se ha definido la arquitectura y componentes apropiados, se ha implementado un prototipo siguiendo dicha propuesta.

Se han analizado distintos trabajos sobre videowalls basados en RPi y se han mejorado, añadiendo más funcionalidades, utilizando reproductores independientes en cada pantalla, y dispositivos de bajo coste. Además, se ha incluido una gestión integral del videowall mediante una aplicación web y, por tanto, multiplataforma.

Como trabajo futuro se pretende realizar evaluaciones objetivas y subjetivas para analizar su idoneidad, aumentar las funcionalidades (p.ej., la visualización de contenido en directo a través de streaming IP basado en RTP [8] o MPEG-DASH). También se pretende añadir multiplexores analógicos al circuito de audio, para tener diferentes equipos de sonido y enviar a cada uno el audio o audios que se deseen, controlables mediante salidas GPIO (General Purpose Input/Output) de las RPi.

\section{AGRADECIMIENTOS}

Los autores quieren agradecer a $\mathrm{M}^{\mathrm{a}}$ José Canet y José Vicente Llario, profesores del Dept. de ingenieria electrónica de la UPV, su ayuda en la realización del circuito electrónico para controlar las salidas de audio.

\section{REFERENCIAS}

[1] A. Jiménez, “Videowall disforme sobre redes IP”, Trabajo Final de Grado, Universitat Politècnica de Catalunya, julio 2016.

[2] PiWall: http://www.piwall.co.uk (ult. acceso mayo 2017).

[3] Video Wall using the Raspberry Pi (ult. acceso mayo 2017). https://www.yodeck.com/news/video-wall-using-raspberry-pi/

[4] GStreamer: open source multimedia framework. https://GStreamer.freedesktop.org/ (último acceso mayo 2017)

[5] Proyecto OMXPlayer, http://omxplayer.sconde.net/ (último acceso mayo 2017)

[6] D. Mills, J. Martin, J. Burbank, and W. Kasch, "NetworkTime Protocol Version 4: Protocol and Algorithms Specification", RFC 5905, June 2010.

[7] M. Montagud, F. Boronat, B. Roig, A. Sapena, "How to perform AMP? Cubic adjustments for improving the QoE”, Computer Communications, Volume 103, 1 May 2017, Pages 61-73, ISSN 0140-3664.

[8] H. Schulzrinne, et al, "RTP: A Transport Protocol for Real-Time Applications", IETF Standard, RFC 3550, July 2003. 